\section{Sobre la llamada revolución psicofarmacológica: el descubrimiento de la clorpromazina y la gestión de la locura}

\author{
On the so-called \\ psychopharmacological \\ revolution: the discovery \\ of chlorpromazine and the \\ management of madness
}

\section{Sandra Caponi $i^{i}$}

'Professora, Departamento de Sociologia e Ciências Políticas/ Universidade Federal de Santa Catarina, campus Trindade. Florianópolis - SC - Brasil

orcid.org/0000-0001-8180-944X

sandracaponi@gmail.com

Recebido em 4 set. 2019.

Aprovado em 13 jan. 2020
CAPONI, Sandra. Sobre la llamada revolución psicofarmacológica: el descubrimiento de la clorpromazina y la gestión de la locura. História, Ciências, Saúde - Manguinhos, Rio de Janeiro, v.28, n.3, jul.-set. 2021, p.661-683.

\section{Resumen}

El artículo analiza la fragilidad y los problemas existentes en la tesis que afirma la existencia de una verdadera revolución en el campo de la psiquiatría biológica, que se habría operado entre 1952 y 1954, con el descubrimiento de la clorpromazina. Para eso se analizan los discursos y las estrategias que posibilitaron el descubrimiento de esta droga que servirá de modelo para la producción de nuevos psicofármacos. Se intenta entender, también, qué es lo que se considera como "eficacia terapéutica" de la droga.

Palabras clave: clorpromazina; historia de la psiquiatría; psicofarmacología; eficacia terapéutica.

\section{Abstract}

This article analyzes the shortcomings and problems of the thesis that a true revolution took place in the field of biological psychiatry between 1952 and 1954 thanks to the discovery of chlorpromazine. To do so, it analyzes the discourses and strategies that led to the discovery of this drug, which became a model for the production of new psychopharmaceuticals. It seeks to understand, also, what is meant by "therapeutic efficacy" with regard to this drug.

Keywords: chlorpromazine; history of psychiatry; psychopharmacology; therapeutic efficacy. 
F n el mes de octubre de 2000, la Asociación de Psiquiatras Americanos (APA) publicó años de actividades. En ese número Lieberman, Golden y Stroup escribieron un artículo denominado "Drugs of the psychopharmacological revolution in clinical psychiatry". Los autores se refieren allí al descubrimiento de los neurolépticos, en los primeros años de 1950, como siendo un momento de verdadera transformación o revolución en el campo de la psiquiatría:

El desarrollo de fármacos antipsicóticos, en la década de 1950, anunció la edad de oro de la psicofarmacología. Su descubrimiento fue comparable al descubrimiento de antibióticos para enfermedades infecciosas, anticonvulsivos para epilepsia y medicamentos antihipertensivos para enfermedades cardiovasculares. Las drogas antipsicóticas pronto se convirtieron en la piedra angular de la farmacopea para el tratamiento de enfermedades psiquiátricas y el entusiasmo por su potencial invadió el campo de la salud mental (Lieberman, Golden, Stroup, 2000, p.1255). ${ }^{1}$

Referencias como estas, donde se habla de la existencia de una verdadera revolución en el campo de la psiquiatría, se repiten desde 1957, cuando se otorga el Albert Lasker Clinical Medical Research Award, hasta nuestros días (Ban, 2007; Baumeister, 2013; Fond, 2013; Healy, 2000; López-Muñoz, Alamo, Cuenca, 2002; Mazana, Pereira, Cabrera, 2012; Shorter, 1997). Vemos en estas referencias, entre muchas otras (Gaudillière, Thoms, 2015; Kunz, 2014; Missa, 2008) la defensa de la tesis según la cual, entre los años 1952 y 1954, se iniciaría un proceso de radical transformación del saber psiquiátrico. Se dirá que ese momento inaugural permitió "una verdadera revolución en el abordaje de los trastornos psiquiátricos" (López-Muñoz, Alamo, Cuenca, 2002).

Esto significa afirmar que los neurolépticos permitieron superar las limitaciones de los tratamientos de alto riesgo que, hasta ese momento, eran utilizados en los hospitales psiquiátricos. De ese modo, drogas como la morfina, aliadas a terapias de choque, a la lobotomía, o a las terapias de contención, podrán ser dejados de lado.

Las referencias a una transformación o revolución operada en el campo de la psiquiatría por la clorpromazina ya aparece en la década de 1957, cuando Henri Laborit, Pierre Deniker, Heinz Lehmann, Nathan Kline y Robert Noce recibieron el prestigioso premio Albert Lasker Clinical Medical Research Award. Los tres primeros, un cirujano y dos psiquiatras, recibieron el premio por el descubrimiento de la clorpromazina, los dos últimos por el descubrimiento de la reserpina. Estos son resumidamente, los motivos presentados por la Fundación Lasker, para otorgar este importante premio:

El trabajo estableció firmemente una nueva fase de tratamiento e investigación psiquiátrica. Demostró a gran escala e incontrovertiblemente que un medicamento puede influir en el curso clínico de las psicosis. Los problemas de la psiquiatría fueron puestos al alcance del laboratorio experimental como nunca antes y el futuro de repente resultó más brillante y prometedor (Lasker Foundation, 1957).

El objetivo de este artículo es mostrar la debilidad de esa tesis, ampliamente difundida, según la cual, el descubrimiento de la clorpromazina, iniciaría una verdadera revolución o ruptura epistemológica en el campo de la psiquiatría biológica. Para eso será necesario 
analizar los discursos y las estrategias de intervención que dieron inicio a la era de los neurolépticos, observando las continuidades y las rupturas que efectivamente ocurrieron en relación a las terapéuticas utilizadas antes del descubrimiento de la clorpromazina (Parada, 2016).

En lo que se refiere a la historia de la clorpromazina, ante la imposibilidad de abarcar toda la literatura existente, tomaré como punto de partida el libro de Judith Swazey (1974) Chlorpromazine in psychiatry: a study of therapeutic innovation. A partir de la lectura de ese libro, propongo iniciar un diálogo, tanto con las fuentes primarias como con los numerosos autores que adoptan una perspectiva crítica en relación al descubrimiento de la clorpromazina (Breggin, 2016; Freitas, Amarante, 2016; Gotszche, 2016; Moncrieff, 2008; Amarante, Pitta, Oliveira, 2017; Whitaker, 2010; Pignarre, 2006; Rose, 2019).

\section{Una narrativa triunfalista}

Cuando analizamos la historia de la psicofarmacología, en general, y la historia de la clorpromazina, en particular, encontramos una y otra vez una referencia que se repite. Diversos autores (Whitaker, 2017; Moncrieff, 2013; Healy, 2000, 2002; Henckes, 2011; Missa, 2006, 2008; López-Muñoz, Alamo, Cuenca, 2002; Gaudillière, Thoms, 2015) insisten en afirmar que la historia más completa hasta hoy existente sobre el descubrimiento de la clorpromazina es el mencionado libro de Judith Swazey (1974).

Este libro revisa los principales textos de referencia, realiza entrevistas con actores que participaron directa o indirectamente del descubrimiento, presenta datos relevantes, traza una cronología histórica muy coherente y pertinente. Sin embargo, parece que se trata de una historia sin problemas, o de una historia cuya temática central gira en torno a la cuestión del precursor.

Swazey (1974) diseña una minuciosa "historia hagiográfica", donde se suceden grandes nombres y grandes conquistas, presentando las ideas que circulaban en un grupo de científicos obsesionados por descubrir un medicamento que, a semejanza de la penicilina, pudiera salvar las vidas de los sujetos condenados a la locura. Relata los caminos metodológicos del descubrimiento, identificando pautas científicamente establecidas que permitieron superar "barreras ideológicas" (p.193) existentes.

Cada capítulo que compone Chlorpromazine in psychiatry se presenta como un paso dado en el camino de una "revolución psicofarmacológica", que lleva a la conquista de una "innovación terapéutica" capaz de imponerse a las visiones ideológicas y retrogradas entonces imperantes. O como afirma la autora, un descubrimiento que permite "romper con las barreras ideológicas" existentes (Swazey, 1974, p.193). Y será como una de esas barreras ideológicas que Swazey piensa al psicoanálisis. Así se refiere la autora al lugar que el psicoanálisis ocupaba en 1950:

Un factor importante fue que la psiquiatría estadounidense a mediados de la década de 1950 estuvo dominada por lo que Armor y Klerman denominaron la ideología psicoterapéutica generada en el transcurso de medio siglo por el psicoanálisis freudiano y por los nuevos movimientos que evolucionaron a partir de los principios de Freud (Swazey, 1974, p.196). 
La autora celebra que, a pesar de estas barreras, el uso de la droga se expandió de tal modo en EEUU, que acabó siendo un tratamiento casi universal. Esta posición de Swazey no es compartida por psicoanalistas como Gladys Swain (1994, p.271), quien destaca la relación históricamente existente entre el uso de neurolépticos y la terapia psicoanalítica. Swain sostiene, sin embargo, que esa relación compleja es poco cuestionada. Afirma: "practicamos quimioterapia y psicoterapia, solo hablamos de psicoterapia, porque es el objeto cultural digno cuando el otro es inmencionable" (p.269).

A diferencia de Swain, Swazey opone lo que denomina "ideología psicoterapéutica" a la somatoterapia, en este último caso, los comportamientos perturbados son considerados, principalmente, como siendo alteraciones funcionales del cerebro. Swazey (1974, p.197) afirma que la psiquiatría biológica y la somatoterapia, defendida por aquellos que adoptaban una posición contraria al psicoanálisis, aunque tuvo ciertas resistencias se impuso con el correr de la década del 1950, cuando "solo algunos fanáticos" se negaban a utilizar la droga.

Retomando los mismos argumentos defendidos por Swazey, Edward Shorter (1997), en su libro A history of psychiatry: from the era of asylum to the age of Prozac, afirma que la clorpromazina inició una revolución en la psiquiatría comparable a la introducción de la penicilina en la medicina general. Una afirmación que repite, en 2001, un psiquiatra del Hospital Lierneux, de Bélgica, entrevistado por Missa (2006): “Para mí el Largactil (nombre comercial de la clorpromazina en Bélgica) es tan importante como la penicilina".

En 1975, un año después de la publicación del libro de Swazey, Deniker escribe un pequeño texto denominado "Qui a inventé les neuroleptiques?", donde se distancia de la perspectiva adoptada por la autora. Deniker afirma que el libro de Swazey es un texto realizado por encargo, financiado por la Academia de Ciencias Americana. En ese momento, la historia del descubrimiento de la clorpromazina no era muy conocida en Francia, excepto por los científicos directamente implicados. Para Deniker, esa historia fue popularizada en 1970 por un breve texto de Ann Caldwell (1970), denominado Origins of psychopharmacology from CPZ to LSD, que estaba fundamentalmente dedicado a la figura de Laborit y a los pesquisadores americanos. Pocos años más tarde la Academia de Ciencias Americana consideró deseable financiar un trabajo mejor documentado, encargándole esa tarea a Judith Swazey, una especialista en historia de la ciencia.

Aun cuando Deniker evalúa que se trata de un estudio bien documentado, señala algunas limitaciones. Afirma que la autora no hace ninguna referencia al concepto de "neuroléptico" y que minimiza el papel del grupo del Hospital Sainte-Anne, coordinado por Jean Delay. Enumera las publicaciones del grupo, las primeras dedicadas al uso de la clorpromazina para la psicosis, afirmando que el texto de Swazey no respeta el orden cronológico de las publicaciones, porque está organizado a partir de las fechas en que los diferentes investigadores recibieron muestras de la droga:

Así, los especialistas que trabajaban regularmente para los laboratorios tuvieron el crédito de una cierta prioridad, aunque su trabajo hubiera aparecido mucho después que otros. De modo que, de las dos investigaciones americanas, la primera carece de la más elemental objetividad (Caldwell, 1970), y la segunda (Swazey, 1974), por la acumulación de elementos 'inéditos' ayudó a dar visibilidad a la industria farmacéutica francesa y americana (Deniker, 1975, p.8). 
Mi discordancia con el trabajo de Swazey está centrada en la falta de cualquier abordaje crítico a los efectos provocados por la clorpromazina. Aunque la autora no deja de hacer mención a los efectos indeseados de la droga, ya bien documentados por Delay y Deniker, prácticamente no hace ninguna referencia a los efectos sedantes profundos ni a la sensación de apatía e indiferencia, sea en relación al mundo o en relación al tratamiento, que caracteriza a los pacientes tratados (Caponi, 2019).

Analizo aquí los argumentos presentados por los ganadores del Lasker Award por el descubrimiento de la clorpromazina: el cirujano Henri Laborit (Laborit, Huguenard, 1952) y los psiquiatras Pierre Deniker (Delay, Deniker, 1952a) y Heinz Lehmann (Lehmann, Hanrahan, 1954).

\section{Sobre la eficacia terapéutica de la clorpromazina}

Judith Swazey (1974) afirma que la clorpromazina fue, como muchos otros fármacos, descubierta por acaso. En realidad, ese medicamento fue inicialmente creado y pensado como un antihistamínico por la empresa farmacéutica Rhône-Poulenc en la década de 1940. Inicialmente, la empresa estaba interesada en estudiar las fenotiazinas para control de helmintos. Aunque esta investigación no tuvo éxito para el objetivo propuesto, algunas de esas fenotiazinas resultaron eficaces como antihistamínicos. Uno de los antihistamínicos estudiados, la prometazina, tenía también propiedades anestésicas y sedantes, y comenzó a ser usada en terapias de sueño. Se trataba de un poderoso antihistamínico, que mostró ser eficaz para el tratamiento de alergias. La trayectoria de la clorpromazina continuó con la observación realizada por Henri Laborit, un médico de la marina francesa que realizaba investigaciones sobre diversos antihistamínicos capaces de ayudar a potencializar la anestesia para realizar cirugías en los soldados. Laborit le solicitó al laboratorio RhônePoulenc el envío de un antihistamínico que fuera más potente que el Phenergan para mejorar las condiciones de las intervenciones quirúrgicas. Entonces, Laborit pudo observar que los pacientes tratados con esa nueva prometazina, la clorpromazina, mostraban un fuerte cambio en su comportamiento, se sentían más tranquilos, calmos y somnolientos, sin perder la conciencia.

La clorpromazina fue utilizada inicialmente por Laborit (1951) para la hibernación artificial, como estabilizador vegetativo. En 1952, Laborit publica el primer artículo sobre el 4560RP. Allí destaca "el desinterés del enfermo por lo que ocurre alrededor de él, llegando a hablar de 'lobotomía farmacológica'”(Missa, 2006, p.255). La molécula conocida con el nombre 4560RP fue testada por diversos equipos que exploraron su eficacia como anestésico, también estudiaron su utilidad para la enfermedad de Parkinson y como tratamiento para el dolor. Laborit afirma que sus estudios habían mostrado que la clorpromazina presentaba "un significativo avance con relación a la prometazina, particularmente por la indiferencia psíquica al dolor" que produce (Laborit, Huguenard, 1952, p.206).

Los estudios realizados por el laboratorio francés Rhône-Poulenc sobre la molécula 2460RP, enviada a Laborit, se habían realizado sobre modelos animales. Estas investigaciones 
no se diferencian de los estudios que, aún hoy, se continúan realizando para testar psicofármacos (Cynowiec, 2017). Las ratas habían sido entrenadas para responder accionando un dispositivo de escape cuando se encontraban expuestas a una amenaza física, como suelo electrificado, por ejemplo. Sin embargo, aun conociendo la estrategia de fuga para evitar la descarga, las ratas que habían sido tratadas con el compuesto 4560RP, que será luego denominado clorpromazina, se mostraban insensibles y completamente incapaces de reaccionar.

Laborit (1951) utilizó esta molécula en pacientes sometidos a algún tipo de cirugía, y comprobó que dejaba a los pacientes en un estado que describió como "hibernación artificial". Un estado semejante al que tienen los animales que deben adaptarse a condiciones extremadamente frías del ambiente. Por ese motivo, y por esa analogía con el fenómeno de la hibernación, muchas veces los médicos intentaban potencializar el efecto de la droga, sumergiendo a los pacientes en hielo, un procedimiento muy utilizado como auxiliar de la anestesia. Laborit llega a sugerir que la clorpromazina podría ser utilizada en el campo de la psiquiatría, en la medida en que permitía lograr efectos semejantes a la lobotomía, permitiendo evitar esa cirugía considerada eficaz pero irreversible. Hoy puede parecer extraño defender un medicamento por ser capaz de inducir lo que se ha descrito como una "lobotomía química", pero, en ese momento, esas eran palabras elogiosas. El inventor de la lobotomía frontal, el neurólogo portugués Egas Moniz, acababa de recibir, en 1949, el premio Nobel de Medicina (Nobel Prize, 1949) por esta cirugía. La clorpromazina había mostrado su capacidad para transformar los pacientes que llegaban agitados y estresados a la sala de cirugía en sujetos calmos, apáticos e indiferentes al procedimiento quirúrgico, como si hubieran realizado una lobotomía.

En el texto de Swazey, la referencia a ese sentimiento de indiferencia aparece de modo indirecto, es mencionado algunas veces, pero nunca es cuestionado o discutido por la autora. Cuando observamos la sucesión cronológica de trabajos publicados sobre clorpromazina, iniciada en 1952, vemos que el primero de ellos fue publicado el 13 de febrero de 1951 por Henri Laborit. Aun cuando estaba centrado en la perspectiva anestesiológica, Laborit subraya un efecto psíquico provocado por la droga, el "desinterés". Ese será el punto de partida para la entrada de la clorpromazina en el campo de la psiquiatría. Los pacientes medicados eran personas que debían permanecer en el espacio cerrado del hospital, y la clorpromazina parecía ser un excelente auxilio para la gestión de las salas de pacientes alterados. Swazey (1974, p.146) simplemente se limita a repetir en algunos momentos del texto que, para algunos observadores, la respuesta psiquiátrica más impresionante de la clorpromazina era "la somnolencia" y la "indiferencia" de los pacientes. Recordemos que, en 1952, Delay y Deniker (1952b, p.115) relataron los efectos psíquicos provocados por el medicamento del siguiente modo:

Del punto de vista psico-fisiológico, en las ratas condicionadas, pudimos notar una indiferencia a los estímulos externos. ... Los efectos psíquicos observados en los pacientes son: somnolencia, pasan la mayor parte del tiempo durmiendo, cuando son despertados permanecen obnubilados. ... En general los enfermos tratados permanecen somnolientos y como indiferentes. Sin embargo, el término 'lobotomía química' propuesto para caracterizar el estado de estos pacientes, no nos parece correcto. 
Esa misma apatía ya había sido relatada por Laborit cuando observaba a sus pacientes tratados con clorpromazina para procedimientos quirúrgicos. Una indiferencia que se manifestaba también en circunstancias ajenas a las cirugías, incluso en circunstancias que podían significar un potencial peligro, tal como había sido observado en los estudios realizados con modelos animales. Según Healy (2002), considerando esta abulia o indiferencia a las amenazas externas, Laborit había recomendado que la clorpromazina se usara para controlar las reacciones de estrés en situaciones límites, como las que se vivían en el campo de batalla. "Siguiendo esta recomendación, se incluyó la clorpromazina en los botiquines médicos de los soldados estadounidenses en la Guerra de Corea" (p.37).

\section{La clorpromazina en el Hospital Sainte-Anne}

Vemos así que la clorpromazina inicia su trayectoria farmacológica como antihistamínico, luego se utiliza como anestésico y solo en tercer lugar ingresa a la sala del hospital psiquiátrico para el tratamiento de pacientes psicóticos. Para entender este uso de la droga, debemos situarnos en el Hospital Psiquiátrico Sainte-Anne de París en 1952. En ese momento el director de ese hospital era Jean Delay, una figura fundamental para la aceptación de los trabajos que bajo su gestión serán realizados por el equipo de Pierre Deniker para el descubrimiento de la clorpromazina. Para Healy, la historia de ese descubrimiento podría haber sido completamente diferente, porque antes de que Deniker o cualquier otra persona afirmara que esta droga podría ayudar a tratar la esquizofrenia, Henri Baruk (1967, p.54) y sus colegas, también del Hospital Sainte-Anne, habían administrado esa droga a animales de laboratorio, concluyendo que producía catatonia experimental y que, en lugar de curar la esquizofrenia, podía llegar a causarla.

Pierre Deniker y su equipo comenzaron a realizar estudios en los pacientes psicóticos internados en el Hospital Sainte-Anne. En 1952, Delay y Deniker (1959) presentaron seis artículos en coautoría. Esos artículos se denominan: "Utilización en terapéutica psiquiátrica de una fenotiazina de acción central electiva", publicado en los Annales Médico-Psychologiques el 26 de mayo de 1952; "Tratamiento de los estados de excitación y de agitación por un método medicamentoso derivado de la hibernoterapia", publicado el 23 de junio de 1952 en la misma revista; "Tratamiento de estados de confusión por la clorpromazina (4560RP)", publicado también en los Annales, el 7 de julio de 1952; "Tratamiento de psicosis por un método neuroléptico derivado de la hibernoterapia (4560RP), utilizado en cura prolongada y continuada", publicado en las Actas del $50^{\circ}$ Congreso de Alienistas y Neurólogos de la Lengua Francesa, el 27 de julio de 1952; "38 casos de psicosis tratada por la cura prolongada y continuada de 4560RP", trabajo publicado en las Actas del Congreso, en la misma fecha; y, finalmente, "Reacciones biológicas observadas en el curso del tratamiento por clorpromazina", tercer trabajo presentado en el mismo congreso (Deniker, 1975, p.9-10).

Esos seis trabajos ponen en evidencia de qué modo el equipo del Hospital Sainte-Anne pensaba la acción del fármaco. Deniker (1975) afirmaba que, para entender la acción de los medicamentos y para poder reconstruir la historia del descubrimiento de un fármaco, existen tres opciones posibles: (1) la vía químico-farmacológica, (2) la vía de la fisiología 
humana, de la anestesiología y reanimación, y (3) la busca de medicamentos psiquiátricos específicos. Las dos primeras vías se refieren específicamente al descubrimiento del fármaco y a la elección del modo de aplicación, esto es, a los estudios (como los realizados por Laborit) referentes a los mecanismos de acción de la droga y sus potencialidades para provocar los efectos deseados, en ese caso, hibernación y anestesia. Según Deniker, esas dos primeras vías son indirectas y no permiten entender el alcance y la importancia de la droga en el campo de la psiquiatría. Considera que solo la tercera vía posibilitó el descubrimiento específico de la acción de la clorpromazina en el campo de la psiquiatría y con ella estaban comprometidos los investigadores del Hospital Sainte-Anne:

Una idea estaba presente en nuestro espíritu, era la posibilidad de descubrir un medicamento específico para el tratamiento de las enfermedades mentales. ... La idea del posible descubrimiento de medicamentos psiquiátricos específicos no era considerada por los especialistas hasta ese momento (Deniker, 1975, p.14).

Entre los estudios presentados en 1952 por Delay y Deniker, será analizado aquí el articulo denominado "38 cas de psychoses traitées par la cure prolongée et continue de 4560RP". En ese texto, Delay y Deniker (1952a) estudian los efectos de la droga en un conjunto de pacientes que fueron divididos en cinco grupos: "excitación psíquica y agitación"; "estados de confusión"; "estados ansiosos y afligidos"; "esquizofrenia" y, finalmente, el grupo compuesto por "delirantes, alucinados y obsesivos".

Como es posible observar, resulta muy difícil hablar de eficacia terapéutica de la droga para una determinada patología, porque el tratamiento no estaba destinado a un tipo de diagnóstico específico sino que se testaba para diversos diagnósticos, entre los cuales existen superposiciones. Existen comportamientos o síntomas que se repiten en diferentes cuadros clínicos, como agitación, excitación, confusión mental. Podemos observar que, en el momento de definir esos diagnósticos, los autores recurrieron a lo que hoy denominaríamos "agrupaciones de síntomas". En el primer caso, aparecen los siguientes comportamientos: accesos maníacos, confusión mental, gran agitación, reacciones agresivas; en el según caso aparecen confusión mental y pensamientos delirantes. Muchos de esos síntomas se repiten en los cuadros definidos como "estados ansiosos y depresivos". En la mayor parte de esos cuadros, el tratamiento preconizado varía entre cinco a 23 días, siendo que en algunos casos hay más de un tratamiento. La dosis utilizada no se altera, manteniéndose entre 50 y $150 \mathrm{mg}$.

En general, los resultados logrados son considerados eficaces en lo que se refiere a la sedación, disminución de la agitación, desaparición de confusión, control de movimientos anormales. Es relatada también la existencia de mejora transitoria. En algunos casos se habla de cura y de salidas del hospital. En relación a los pacientes del tercer grupo, la mayor parte no demuestra recuperación del cuadro de depresión, aunque los autores sí observan progreso en relación a los síntomas de manía y agitación. En el caso de los pacientes esquizofrénicos, no hay ninguna mención a alucinaciones o delirios, y los resultados relatados son: (paciente 27) desaparición de confusión y ansiedad; (paciente 28) sedación de la agitación; (paciente 29) desaparición de movimientos anormales; (paciente 30) mejora de 
agitación y excitación; (paciente 31) remisión de la agitación; (paciente 32) calma y mejor contacto (Delay, Deniker, 1952a, p.506-508). En relación al último grupo, se observa que se repiten los mismos efectos terapéuticos ya relatados: sedación y control de la agitación, pero se habla de aumento inicial de las alucinaciones. Vale resaltar que muchos de estos pacientes ya habían sido sometidos, diversas veces, a electrochoque, insulinoterapia y otros tratamientos de choque.

El estudio muestra que la clorpromazina no fue utilizada exclusivamente para casos de esquizofrenia, como ocurrirá posteriormente. Su función terapéutica principal parecía reducirse a controlar la agitación y la ansiedad, provocando sedación. La droga no parece ser eficaz para la depresión, siendo eficaz para casos de agitación motora y manía. "Los neurolépticos son efectivos contra la manía y, raramente, pueden inducir cierto grado de depresión (como la reserpina)" (Deniker, 1990, p.86).

Algunos años más tarde, en 1956, Deniker y Delay publicaron, en la revista L'Encéphale, un artículo de evaluación retrospectiva de los efectos del uso de la clorpromazina en enfermos mentales atendidos en una clínica entre 1952 y 1955. Ese acompañamiento fue realizado con un total de trecientos informes de pacientes, internados o en atendimiento ambulatorio que recibieron clorpromazina por un período de casi cuatro años. Los autores afirman que es difícil establecer una comparación con otras investigaciones, dada la ambigüedad y la discrepancia entre las clasificaciones nosológicas utilizadas en esos estudios. El trabajo realizado por Deniker y Delay (1956, p.528) está centrado en tres puntos: la acción de la clorpromazina en las psicosis agudas; la acción de la droga en la depresión; y el papel de las curas prolongadas posibilitadas por el uso de la clorpromazina en el tratamiento de psicosis crónicas.

Consideran que en relación al primer grupo, psicosis agudas, las expectativas iniciales sobre la eficacia de la clorpromazina pudieron confirmarse, constituyendo una verdadera prueba de los rápidos efectos de la droga. El estudio indica que un gran número de pacientes fue curado y recibió alta. Registran que la duración media de hospitalización es menor comparativamente a los pacientes que utilizaron electrochoque. Otro dato presentado es que el tiempo de hospitalización de las mujeres es mayor que el de los hombres. En relación al diagnóstico de depresión afirman que "los síndromes depresivos simples, así como las depresiones dichas de reacción, pueden reaccionar favorablemente al tratamiento exclusivo con neurolépticos" (Deniker, Delay, 1956, p.532).

Por fin, en relación a los tratamientos prolongados de psicosis crónica, el estudio se realizó sobre un total de 116 pacientes, incluyendo cuadros de esquizofrenia. Los autores evalúan el uso de la clorpromazina separada o asociada a electrochoque y a otras drogas, y también a la lobotomía, mostrando resultados que consideran positivos. Destacan la importancia de los tratamientos ambulatorios por vía oral que permiten dar continuidad a la medicación después de la salida del manicomio (Deniker, Delay, 1956, p.533).

Este texto nos permite entender qué es lo que estos autores consideran como siendo un resultado eficaz y exitoso del punto de vista terapéutico. Afirman que la clorpromazina no reduce las alucinaciones ni los delirios: 
La instalación de un estado de indiferencia y desinterés con relación al delirio es lo que parece constituir la originalidad de ese tratamiento. El problema de la desaparición real de las alucinaciones o de la actividad delirante es más complejo. A pesar de que en algunos casos parecen detenerse, es frecuente que el enfermo conserve la creencia en el delirio. Continúa viviendo con la existencia de 'ideas delirantes' en relación a las cuales ya no manifiesta preocupación después del tratamiento, sino indiferencia (Deniker, Delay, 1956, p.535).

Podemos observar, una vez más, que la cuestión de la indiferencia (sea indiferencia al mundo, al medio o a los síntomas de la enfermedad) parece ser la llave de comprensión del modo como la droga efectivamente actúa. Esto es, el sentimiento de indiferencia parece estar directamente asociado a lo que se considera como eficacia terapéutica de la droga. De modo que, aunque los pacientes tratados con clorpromazina continuarán teniendo alucinaciones, parecen ser indiferentes en relación a esas visiones.

Delay y Deniker relataban en sus trabajos la eficacia de la clorpromazina para calmar a los pacientes en las salas de enfermos psicóticos. Algunos estudios realizados posteriormente en el Hospital Sainte-Anne y otros hospitales relatan que los pacientes afirmaban haber dejado de escuchar voces que les pedían volver a sus domicilios y tenían la sensación de haber recuperado cierta normalidad (Healy, 2000). Sin embargo, el suceso de la clorpromazina no fue tan inmediato como podría imaginarse. Aunque Delay y Deniker se preocuparan por divulgar sus observaciones en revistas médicas de prestigio y en congresos internacionales, esas investigaciones no tuvieron inmediatamente el impacto esperado. Muchos psiquiatras sostenían que el tratamiento de la psicosis era imposible y que solo se trataba de un sedante bastante efectivo. De acuerdo a Healy (2002, p.92), "Henri Ey consideró que la clorpromazina era útil en la administración de terapias del sueño, pero no iría más allá. Incluso dentro de Sainte-Anne, el nuevo tratamiento no fue adoptado inmediatamente por todos". Quizás existían fuertes razones para ese escepticismo.

Leyendo los textos de Deniker podemos observar claramente que su trabajo llamaba a ser escépticos y cautelosos. Ocurre que el descubridor de la clorpromazina se enfrentará rápidamente a los efectos adversos de la droga, él insistirá en la necesidad de tener cautela extrema, destacando que el uso de clorpromazina podía generar un estado denominado discinesia tardía en los pacientes. Los pacientes presentaban movimientos involuntarios en sus miembros y en su rostro, acompañados de rigidez muscular y temblores. Este efecto adverso de clorpromazina fue relatado por Delay y Deniker ya en 1956, en un artículo denominado "Extrapyramidal movement disorders during chlorpromazine and reserpine therapy; clinical and electromyographic studies" (Delay, Deniker, Bourguignon, 1956), publicado en la revista L'Encéphale. Este es un tema que Deniker retoma en diferentes momentos, inclusive en un artículo publicado casi 35 años más tarde bajo el nombre "From chlorpromazine to tardive dyskinesia: brief history of the neuroleptics" (Deniker, 1989).

Por otra parte, aunque los efectos colaterales de la droga se mostraban devastadores, el efecto deseado de tranquilizar a los pacientes manteniéndolos despiertos, parecía haber sido logrado. Los estudios realizados por Deniker mostraban que la clorpromazina era mucho más que un simple tranquilizante. Missa resume los efectos de la droga presentados en 1952 por Delay y Deniker: 
El efecto psicológico más constante era la inercia y el desinterés. Las excitaciones del mundo exterior parecían llegar de un modo amortizado. Ellos responden a los estímulos con un ligero retardo, con un tono neutro e indiferente, los ojos bajos. Hundidos en una dulce indiferencia, parecen separados del ambiente por un tabique invisible (Missa, 2006, p.259).

\section{Heinz Lehmann y la indiferencia emocional}

Como ya fue mencionado, en 1957 la Asociación Americana de Salud Pública otorgó el Lasker Award a Heinz E. Lehmann por la introducción de la clorpromazina en el campo de la psiquiatría. Particularmente por la publicación, en 1954, del artículo en coautoría con G.E. Hanrahan denominado "Chlorpromazine: new inhibiting agent for psychomotor excitement and manic states", en los Archives of Neurology and Psychiatry (Lehmann, Hanrahan, 1954).

El Lasker Award, fue dado a Lehmann en reconocimiento a la elaboración de un modelo clínico para aplicación de la clorpromazina, explicitando los beneficios y las dificultades de la droga y minimizando sus riesgos. Según informa la página de Lasker Award, el premio fue otorgado porque:

En su primera publicación importante sobre lo tema, el Dr. Lehmann fue capaz de delinear las pautas clínicas tan claramente, describir los resultados con tanta precisión y evaluar los peligros con tanta franqueza que, solamente con ese documento, cualquiera otro psiquiatra estaría en condiciones de aplicar esa medicación con confianza y seguridad. Notó no solo el efecto en el paciente individual, sino también el efecto general en los grupos y, especialmente, en las salas alteradas (Lasker Foundation, 1957).

Aunque el premio fue otorgado por el "uso de la clorpromazina para el tratamiento de esquizofrenia", sin embargo, Lehmann (1955) solo se referirá a los pacientes con esquizofrenia un año más tarde, en el artículo "Chlopromazine in psychiatric conditions". Los dos textos de Lehmann aquí citados, permiten entender cuáles eran los elementos que consideraba relevantes para hablar de la eficacia terapéutica de la clorpromazina. En una entrevista realizada por Swazey (1974), poco antes de su muerte, Lehmann decía que la clorpromazina revolucionó el campo de la psiquiatría haciendo de esta una verdadera especialidad médica, capaz de tratar a las enfermedades mentales como cualquier enfermedad, esto es, con medicamentos (Lehmann, Swazey, 1974, p.157).

El primer estudio de Lehmann se apoya en una experiencia realizada en el Hospital Protestante de Verdun, donde fueron tratados con clorpromazina 71 pacientes psiquiátricos con edades entre 18 y 82 años, durante un período de cuatro meses. Ese estudio fue realizado con pacientes con diagnósticos de manía aguda y crónica, esquizofrénicos, pacientes con demencia senil, deficiencia mental y otros que sufrieron lobotomía.

Los pacientes que mostraron una recuperación mayor fueron quienes habían sido tratados por más tiempo y con dosis más altas de clorpromazina, algunos de los cuales habían llegado a recibir $800 \mathrm{mg}$, mucho más que los 150mg que Deniker preconizaba como dosis máxima. Para los autores, el estudio muestra una acción efectiva de la droga para 
"el control sintomático" de estados de excitación, sin necesidad de utilizar otros tratamientos auxiliares, como el electrochoque. La Figura 1 presenta una tabla que condensa los resultados del primer estudio realizado por Lehmann.

Therapeutic Results with Chlorpromasine*

\begin{tabular}{|c|c|c|c|c|c|c|c|}
\hline & Averted & $\begin{array}{c}\text { Re- } \\
\text { covered }\end{array}$ & $\begin{array}{l}\text { Much } \\
\text { Iro- } \\
\text { proved }\end{array}$ & $\begin{array}{c}\text { Im- } \\
\text { proved }\end{array}$ & $\begin{array}{c}\text { Ud. } \\
\text { cbanged }\end{array}$ & $\begin{array}{c}\text { stiu } \\
\text { Under } \\
\text { Treat. } \\
\text { mest }\end{array}$ & Total \\
\hline Beblzopbrenf & 2 & .. & 3 & 。 & 7 & 1 & 22 \\
\hline Bebizontreetive............................ & $\ddot{*}$ & .. & 1 & 2 & .. & .. & $\mathbf{3}$ \\
\hline Menle, acute............................ & 2 & 8 & 2 & 7 & .. & $\mathbf{s}$ & $n$ \\
\hline Manle, chronie.......................... & .. & 4 & 1 & 3 & 1 & $\mathbf{3}$ & 12 \\
\hline Psychoneurotte......................... & .. & 1 & .. & 1 & 2 & .. & 1 \\
\hline Postlobotomy.......................... & .. & $*$ & .. & 2 & 1 & 1 & 4 \\
\hline Meotal dedeleney...................... & .. & $*$ & $\cdots$ & .. & 2 & .. & 1 \\
\hline Seollo paycbosis............................ & $\ddot{z}$ & $\ddot{z}$ & $\ddot{-}$ & 3 & $\ddot{*}$ & $\ddot{z}$ & 3 \\
\hline Total.................................. & 4 & $\overline{\mathbf{1 3}}$ & $\overrightarrow{7}$ & $\overline{27}$ & $\overline{12}$ & $\overline{8}$ & $\bar{n}$ \\
\hline
\end{tabular}

- Averted means arrest of immibent paychotic attack durloz prodromal stage; recovered, austoloed cessa thon of symptoms witbin 40 days; much lmproved, reduction of symptorns to tbo point wbero the patient is able to leave hospltal witbin 10 deys; improved, sustained lesseblez of symptome witblo 10 days but patient unable
to leavo bospital.

Figura 1: Resultados terapéuticos con clorpromazina (Lehmann, Hanrahan, 1954, p.233)

La tabla muestra que, aun cuando Lehmann había recibido el Premio Lasker por descubrir el efecto terapéutico de la clorpromazina para la esquizofrenia, su primer estudio parecía indicar que ese efecto no existía. Como afirman los autores:

La mayoría de los pacientes que 'no' mostraron mejora duradera con el fármaco eran esquizofrénicos crónicos que presentaron un deterioro considerable. No observamos una influencia directa de la droga en los sistemas delirantes ni en los fenómenos alucinatorios (Lehmann, Hanrahan, 1954, p.232).

El efecto de la clorpromazina en el tratamiento de pacientes con diagnóstico de esquizofrenia fue el tema del estudio publicado por Lehmann un año más tarde. Allí dirá, contradiciendo los resultados presentados en el primer estudio, que la clorpromazina efectivamente podía revertir algunos casos de esquizofrenia. En la entrevista de Judith Swazey a Lehmann, él dirá que llegó a esa conclusión después de observar a un grupo de pacientes esquizofrénicos que participaron del primer estudio y que accidentalmente habían recibido la medicación por más tiempo. Esos pacientes parecían haber mejorado.

En ese momento, pensamos que solo estábamos tratando estados excitados con la clorpromazina y atribuimos la mejora que mostraron los esquizofrénicos a ese efecto de la droga. Pero, tres meses después de finalizado el ensayo, descubrimos que algunos de los esquizofrénicos crónicos habían continuado recibiendo, accidentalmente, grandes dosis de clorpromazina. Increíblemente para nosotros, cuatro o cinco de esos pacientes estaban mejorando. Nadie creía que una píldora pudiese causar la remisión de la esquizofrenia, y parecía que estábamos logrando mejores resultados que con los paranoicos crónicos, el grupo más refractario a la readaptación (Lehmann, Swazey, 1974, p.157). 
En esa segunda investigación se repite la misma estrategia utilizada en el estudio anterior. La observación fue realizada con pacientes en diferentes estados clínicos, incluyendo manía, depresión, esquizofrenia y pacientes que sufrieron lobotomía. Dentro de esa variedad de casos, se observa a los pacientes entre los cuales la droga presentó mejores efectos de control de síntomas, argumentando que la clorpromazina era la terapéutica apropiada para esas enfermedades. En el estudio de 1955, dirá que la clorpromazina se mostró eficaz para garantizar la remisión de síntomas de esquizofrenia (Lehmann, 1955).

Así, en la entrevista realizada por Swazey, ya mencionada, Lehmann explicaba que su interés no se limitaba a usar el fármaco para calmar pacientes agitados, independientemente de cual fuera el diagnóstico, sino que, en los estudios realizados en 1954 y en 1955, perseguía el ambicioso proyecto de encontrar las bases psicofarmacológicas de la esquizofrenia. Sabemos que ese objetivo no fue alcanzado por Lehmann ni por ningún otro psiquiatra y que, aún hoy, casi cincuenta años después, la busca por el "Santo Grial" continúa. Así Lehmann presentaba su trabajo en 1974:

Entonces, decidí probar clorpromazina. Como no teníamos recursos para nuestras investigaciones en 1953, y solo unos seis médicos para 1.600 pacientes, utilizamos parte de nuestro propio dinero para investigar, como una especie de hobby. Estábamos interesados en encontrar una base psicofisiológica para apoyar el diagnóstico clínico psiquiátrico. Aún estoy buscando por ese 'Santo Grial' (Lehmann, Swazey, 1974, p.155).

Lehmann defiende la existencia de una revolución provocada por la clorpromazina en el campo de la psiquiatría por dos motivos: (1) la clorpromazina le dio a la psiquiatría un instrumento que le permitió aproximarse a otras ramas de la medicina. Aun cuando la base psicofisiológica de las enfermedades mentales fuera desconocida, la clorpromazina se presentaba como un medicamento capaz de revertir los síntomas (y en algunos casos curar) las enfermedades mentales, del mismo modo que un medicamento podía curar una patología biológica; (2) la clorpromazina permitía que los enfermos pudieran salir del hospital y volver a sus domicilios, iniciando el servicio ambulatorio.

En la tabla presentada en la Figura 1, vemos que existe una diferencia entre las categorías "mucho mejor" y "mejor". En el primer caso, se habla de una reducción de los síntomas después de cuarenta días, permitiendo que el paciente salga del hospital. En el segundo, se habla de una disminución sostenida de síntomas en menos de cuarenta días, "pero el paciente no puede abandonar el hospital". Esa era una distinción relevante para Lehmann, porque la clorpromazina permitía la gestión ambulatoria de pacientes que continuarían usando medicación fuera del hospital psiquiátrico.

El texto de Lehmann y Hanrahan (1954, p.234) finaliza con cinco preguntas destinadas a evaluar la incorporación de la clorpromazina en la lista de agentes terapéuticos en el campo de la psiquiatría, de tal modo que se pueda dejar registro de su valor y de su legitimidad. Las preguntas son:

(a) ¿Controla los síntomas tan bien o mejor que otros tratamientos establecidos?

(b) ¿Puede ser favorablemente comparado en relación a la facilidad de administración y a los efectos secundarios desagradables? 
(c) ¿Hay menos riesgo asociado a su uso?

(d) ¿Mejora la condición patológica subyacente?

(e) ¿Permite una relación psicoterapéutica a largo plazo?

En relación a la primera pregunta, Lehmann afirma que con la prescripción de clorpromazina los pacientes maníacos y agitados se recuperaron y disminuyeron los síntomas de la enfermedad. Sin embargo, para poder hablar de la eficacia terapéutica del fármaco, sería necesario definir cuáles eran los síntomas controlados. Lehmann observa un índice mayor de recuperación en la psicosis maníaca, sin especificar los síntomas ni enunciar de qué modo era realizado el diagnóstico. Cabe recordar que el Manual de Diagnóstico y Estadística de Enfermedades Mentales (DSM I) había sido publicado dos años antes, en 1952, y que ese manual de la Asociación de Psiquiatras Americanos definía los síntomas de psicosis maníaco-depresiva de tipo maníaco, como:

Este grupo se caracteriza por euforia o irritabilidad, con exceso de locuacidad, vuelo de ideas y aumento de la actividad motora. Pueden ocurrir episodios de depresión transitorios, frecuentemente momentáneos, pero no alteran la clasificación del tipo 'reacción maníaca' (APA, 1952, p.25).

Si consideramos ese diagnóstico, es fácil imaginar que el efecto sintomático deseado de la droga no será otro que conseguir cierta calma, limitación de la locuacidad, desaceleración psicomotora, control de la irritabilidad. Es en esa dirección que son definidos los parámetros de lo que debe ser considerado un efecto terapéutico eficaz, para verificar si la droga está actuando satisfactoriamente. Tal como enuncian los autores del estudio:

El objetivo es producir un estado de atraso motor, indiferencia emocional y somnolencia, la dosis debe ser aumentada a medida que se desarrolla la tolerancia. Después de la primera semana de tratamiento, los pacientes permanecen lentos, pero tienen menos sueño. En la mayoría de los casos, no fue necesario superar una dosis de $800 \mathrm{mg}$ día, mientras que algunos pacientes pueden requerir solamente 100mg o menos, diariamente. El tratamiento puede durar de tres días a cuatro semanas (Lehmann, Hanrahan, 1954, p.229).

En relación a la segunda pregunta, Lehmann presenta una serie de procedimientos para la facilitar la aplicación de la clorpromazina. Ese es uno de los motivos destacados cuando se le otorga el Lasker Award, por haber delineado claramente las pautas para aplicación de la droga, a través de 13 pasos que indican la forma de uso, la dosis, la dieta, el descanso exigido etc.

Sobre la tercera pregunta, el premio también destacaba la capacidad de Lehmann para evaluar los peligros del uso de la clorpromazina con franqueza, auxiliando otros psiquiatras a conocer las condiciones para aplicar esa medicación con confianza y seguridad. Lehmann no parece desconocer ni desconsiderar los efectos adversos de la droga. Relata que los pacientes se volvieron apáticos, poco dispuestos, menos "alertas", y describe el "vacío de expresión" que observa en sus rostros. Afirma, también, que muchos se lamentaban de que la clorpromazina los hacía sentir "vacíos", que les desagrada el tratamiento, relatando la 
somnolencia y debilidad que acompañaba el uso de la droga. Del mismo modo que Deniker, Lehmann también observa la existencia de síntomas de Parkinson en los pacientes tratados con clorpromazina, aunque afirma que esos síntomas podían ser revertidos después de un cierto tiempo. Sin embargo, y a pesar de haber enumerado todos estos problemas vinculados al uso de la clorpromazina, Lehmann afirma:

Los pacientes que reciben grandes dosis de clorpromazina exhiben retardo motor definido por una marcha inestable, mientras la expresión facial se vuelve bastante rígida y la apariencia general se asemeja a la del parkinsonismo, pero sin rigidez muscular. Con dosis menores, esos fenómenos son menos pronunciados. Generalmente hay somnolencia acentuada, que puede aumentar a punto de adormecer. Esos síntomas se evidencian en la declaración de los pacientes de que ellos sienten frío, somnolencia, desmayo y debilidad (Lehmann, Hanrahan, 1954, p.228).

Agrega que: "A algunos pacientes no les gusta el tratamiento y se lamentan de la somnolencia y debilidad provocada. Algunos afirman que se sienten 'agotados', como después de una enfermedad exhaustiva, una queja que realmente está de acuerdo con la apariencia que presentan!" (Lehmann, Hanrahan, 1954, p.230).

Por fin, Lehmann se pregunta si la droga "mejora la condición patológica subyacente" (Lehmann, Hanrahan, 1954, p.234). Aparece, entonces, la analogía entre los procedimientos terapéuticos utilizados por la medicina y los utilizados por la psiquiatría. Sabemos que es por su identificación con las explicaciones médicas que la psiquiatría conseguirá finalmente su legitimidad epistemológica y su prestigio (Foucault, 2003). Surgen, así, los esfuerzos para mostrar que la clorpromazina es un medicamento idéntico a cualquier otro, por ejemplo a la penicilina. Un medicamento capaz de actuar y de revertir los procesos biológicos que subyacen a la patología, aun cuando se desconozcan los mecanismos de acción de la droga:

El modo de acción de la clorpromazina aún no se ha establecido. Se han avanzado varias teorías. Se ha supuesto que el fármaco funciona a través de un efecto sobre el metabolismo general al reducir los requisitos de $\mathrm{O}_{2}$ celular. No hemos podido confirmar los hallazgos de Delay y Deniker de que el metabolismo basal se reduce después de la administración de clorpromazina.... Por lo tanto, no nos sentimos justificados en este momento a afirmar que el medicamento exhibe sus efectos específicos a través de las glándulas suprarrenales o cualquier otra glándula endocrina (Lehmann, Hanrahan, 1954, p.236).

Frente al desconocimiento de los procesos biológicos subyacentes, se trata de definir la mejor forma de administración de la droga, los efectos colaterales, la dosis correcta, su acción sobre los síntomas. Desconocidas las bases biológicas de la manía o de la esquizofrenia, la eficacia de la clorpromazina parecía limitarse al efecto de sedación con conciencia ya relatado por los estudios anteriores, sin que disminuyan los delirios o alucinaciones. Es así que, en 1954, Lehmann se refiere al mecanismo de acción de la clorpromazina en los pacientes tratados:

La clorpromazina tiene un efecto inhibitorio pronunciado de ciertas funciones del sistema nervioso central. Los pacientes que reciben el medicamento se vuelven 
letárgicos. Pacientes maníacos generalmente no se oponen al reposo y permanecen en sus camas y pacientes que presentaban problemas de manejo se vuelven tratables (administrables). El comportamiento agresivo e interferente cesa casi enteramente. Los pacientes en tratamiento demuestran falta de interés espontáneo en el ambiente, se puede acceder a ellos con facilidad y, generalmente, responden de manera inmediata y relevante a preguntas, aun después de haber sido despertados de su sueño. Como Delay y Deniker los describieron, tienden a permanecer en silencio e inmóviles cuando se los deja solos y a responder a las preguntas en un tono lento y monótono (Lehmann, Hanrahan, 1954, p.230).

Finalmente, la última pregunta se refiere a la capacidad de la droga de "permitir una relación psicoterapéutica a largo plazo", una cuestión que es, al mismo tiempo, epistemológica y política. Se trata de saber si la droga permite que se establezca un vínculo terapéutico con el paciente. Sin embargo, todas las analogías con las intervenciones de choque realizadas en el texto parecen negar esa posibilidad. Aun así, los autores consideran que la clorpromazina podría ayudar a crear un vínculo psicoterapéutico. Como reconoce el propio Lehmann: "Muchos de nosotros, en los últimos años, hemos perdido de vista nuestra tarea esencial de comprender nuestros pacientes, ya que los sometemos a una secuencia de coma, conmociones, convulsiones, confusión y amnesia, que los incapacita para que se relacionen con el psiquiatra de una manera consistente y significativa" (Lehmann, Hanrahan, 1954, p.234).

Podemos preguntar: ¿de qué modo la clorpromazina podría auxiliar a establecer un tipo de vínculo terapéutico basado en la empatía, antes imposible de realizar con los tratamientos de choque? Recordemos que, aun cuando Lehmann destaca las diferencias entre los neurolépticos y las intervenciones anteriormente utilizadas, como el electrochoque (Lehmann, Hanrahan, 1954, p.234), no deja de asociar el uso de la clorpromazina con la lobotomía, tal como lo hiciera Laborit. Así, en 1955, afirma que "en el tratamiento del dolor en casos terminales de cáncer, la clorpromazina puede ser un substituto farmacológico de la lobotomía" (Lehmann, 1955, p.97). Aunque se refiere a sedación para dolores de cáncer, las elevadas dosis preconizadas en los tratamientos de manía y esquizofrenia, 800mg, podrían provocar efectos semejantes a la lobotomía química. Ese dato parece indicar que difícilmente el paciente tendría condiciones para iniciar un proceso de psicoterapia o de terapia por la palabra. Aun sin llegar a la identificación con la lobotomía, las descripciones de Lehmann aquí reproducidas referidas a letargia, somnolencia, vacío de expresión, retardo motor definido, marcha inestable, rigidez de la expresión facial, rigidez motora, movimientos parkinsonianos, producidas por el uso de clorpromazina, parecerían indicar que la capacidad de verbalización y reflexión del paciente se encuentra claramente comprometida, aunque sea capaz de dar respuestas simples a preguntas puntuales.

\section{Una sala tranquila}

Jean Noel Missa reproduce una extensa entrevista realizada en 1999 con una enfermera que desarrolló sus actividades en un hospital psiquiátrico de Canadá. Ella analiza su vínculo terapéutico con los pacientes antes y después de la introducción de los neurolépticos en 
la sala del hospital psiquiátrico en la cual trabajaba. Recuerda que antes ellos bailaban y cantaban, y describe lo que observa en su día a día:

Con los enfermos, antes de la era de los neurolépticos, se podía hablar. Pasé años escuchando a los enfermos. Una paciente venía, aproximaba un banco a mi lado y empezaba a contar su historia. Yo lo escuchaba. Ahora tenemos gente que babosea, que tiembla y tiembla. Pienso que esos medicamentos psicotrópicos los dejan en un estado aterrador. Lo que ellos experimentan con los psicotrópicos, lo que pasa en sus cabezas es aterrador. Los enfermos cambiaron a causa de esos medicamentos, ya no son más los mismos. Prefiero ver los enfermos vivos, aun cuando deliren. Ahora parecen robots (Missa, 2006, p.299).

Lehmann, al contrario, observa esa pasividad de modo positivo. Considera que el uso de la clorpromazina produce un tipo de sedación diferente de la provocada por los barbitúricos o por los tratamientos de choque. Afirma que el efecto terapéutico deseado parecía haber sido alcanzado. Aun cuando las alucinaciones persistieran, el enfermo podía convivir mejor con ellas, ya no se oían gritos en los hospitales y el objetivo de controlar la excitación psicomotora, los estados de manía y los ataques psicóticos de los esquizofrénicos parecía haber sido logrado.

Como afirma un psiquiatra entrevistado por Missa (2006, p.297) al recordar su trabajo en fines de la década de 1950: "El Lagartil era eficiente, pero no curaba. No más que hoy. ... El paciente estaba mejor. Estaba calmo y disciplinado, aunque continuase con sus alucinaciones". Como Lehmann (1955) afirmaba, la clorpromazina permitía el manejo de "problemas de comportamiento" de pacientes psicóticos crónicos. Retomando el texto de Lehmann de 1955, podemos entender mejor que es lo que este premiado psiquiatra entiende por efectividad terapéutica del fármaco:

La clorpromazina puede ser usada como último recurso cuando otras medidas fallaron. El personal de enfermería luego aprendió a apreciar sus efectos favorables, y la introducción del fármaco realmente alteró el aspecto del tratamiento agudo y de las salas del hospital psiquiátrico, por la reducción considerable del ruido y de la confusión, que antes estaban asociados al gerenciamiento (management) de los pacientes perturbados (Lehmann, 1955, p.92).

La idea de que la clorpromazina había llevado calma y sosiego al hospital psiquiátrico es un tópico incansablemente repetido por los psiquiatras a lo largo de los años 1950 y 1960. Así se describía, en 1964, la transformación de un hospital psiquiátrico americano:

¿Te acuerdas de las lobotomías, los vidrios rotos, los gritos sub-humanos en la noche, las ventanas bajo las cuales no se podía caminar, para evitar que excretas de algún tipo caigan sobre ti? ¿Recuerdas el interminable bloqueo y desbloqueo de puertas? ¿Recuerdas a los pacientes maltratados y a los asistentes maltratados? (Swazey, 1974, p.218).

Después de la clorpromazina las intervenciones psiquiátricas ya no se limitaban a los chalecos de fuerza o al electrochoque, ahora se podría tratar la locura con un fármaco, del mismo modo que en cualquier otra rama de la medicina, aunque las intervenciones de 
choques no desaparecieran. Así, en 1953, por referencia al compuesto 4560RP, se afirmaba: "Es un hecho incontestable que gracias a este derivado de las fenotiazinas hemos podido liberar de la camisa de fuerza a todos nuestros sujetos y que la calma obtenida en el pabellón de agitados es impresionante" (Parada, 2016, p.148).

Una y otra vez se hablará del efecto "revolucionario" que la clorpromazina ha tenido en las salas de los hospitales psiquiátricos y en el comportamiento de los pacientes, ahora silenciosos e indiferentes, tal como podemos observar en este relato de 1955:

En los hospitales estadounidenses, la atmósfera de las salas alteradas se ha revolucionado por completo. Los pacientes ahora permanecen vestidos; ellos están callados; no se molestan entre sí; se conforman a las convenciones, se interesan en su aspecto personal y en la apariencia de las salas (Overholser, 1956, p.199).

Siguiendo esa misma línea argumentativa, Judith Swazey (1974, p.220) relata que:

Junto a los efectos de la clorpromazina sobre el comportamiento perturbado, los hospitales de todo el país informaron reducciones significativas en la destrucción de pertenencias personales y mobiliario del hospital, disminución de accidentes y lesiones de pacientes y menor necesidad de utilizar restricciones sedativas, físicas o aislamiento para controlarlos.

La transformación del hospital psiquiátrico tenía implicaciones profundas. Con un medicamento considerado eficaz para el tratamiento de psicosis y, al mismo tiempo, con pacientes y salas más calmas, la psiquiatría finalmente podría conquistar el deseado respeto profesional de sus colegas del campo de la medicina. Un respeto que la psiquiatría parecía haber perdido por la sucesión de intervenciones y tecnologías coercitivas utilizadas en los manicomios. Finalmente parecía posible hablar de la reorganización de la gestión de pacientes perturbados.

Esa nueva gestión no solo implica la reorganización del espacio del manicomio, con salas más tranquilas y calmas, sino también la gestión de la locura fuera del hospital. Así, en el libro denominado Toucher le cerveau, changer l'esprit, Carlos Parada (2016) destaca que la clorpromazina se mostró eficaz, igualmente y con la misma intensidad, tanto para la gestión del espacio interno del hospital, como para la administración de los pacientes ambulatorios. Las intervenciones, poco a poco, dejaron de ser momentáneas, puntuales y esporádicas, como con el electrochoque, para acompañar al paciente a lo largo de su vida, tanto fuera como dentro del hospital. Se inicia así, un proceso de cronificación de la enfermedad mental que exige la continuidad del tratamiento a lo largo de toda la vida del paciente (p.157).

\section{Consideraciones finales}

La clorpromazina no solo permite llevar calma a las salas agitadas de los hospitales psiquiátricos, permite también que algunos de los pacientes tratados con neurolépticos puedan ser considerados menos agitados y más calmos y puedan volver, al menos por 
un tiempo, a sus domicilios. Esos pacientes, ahora calmos e indiferentes, permanecen vinculados al psiquiatra para la renovación y ajuste de las dosis de la medicación.

Desde 1956 (Delay, Deniker, Bourguignon, 1956), se sabe que esta pretendida tecnología revolucionaria de gestión de la locura, este tranquilizante mayor, llamado también de ataráxico, por referencia a la indiferencia o ataraxia que provoca, deja marcas indelebles en el cuerpo de los pacientes. No se trata solo de efectos colaterales indeseados, un problema que comparte con muchos otros psicofármacos, se trata de algo más, de una droga que al mismo tiempo que calma la agitación y la agresividad, produce síndromes que son inherentes a su acción. En la medida en que, como ya lo identificara Deniker en 1956, produce síndromes extra piramidales y vegetativos, inseparables de la acción de la droga. Tal como afirmaba Henri Baruk (1972, p.54):

La naturaleza del síndrome neurológico provocada por los neurolépticos se interpreta de formas diferentes. Delay y Deniker consideran el síndrome neurológico como constantemente asociado a la acción de los medicamentos. Ciertos autores consideran que la obtención del síndrome neurológico es necesaria para determinar los efectos psíquicos de la droga. En otro sentido, los trabajos de Baruk y Berges han mostrado, por primera vez, inicialmente con la clorpromazina y luego con otros neurolépticos, la producción de catatonia experimental, esto es de trastornos psicomotores asociados al uso de la droga.

Sabemos que los neurolépticos están directamente asociados a síntomas de Parkinson y que producen reacciones adversas severas, sin embargo, el uso de antipsicóticos clásicos o atípicos no ha dejado de crecer en las últimas décadas. De lo dicho hasta aquí, podemos concluir que la persistencia del uso de los neurolépticos, a pesar del conocimiento exhaustivo de los daños que produce, se debe a que este medicamento cumple exactamente con los objetivos para los cuales había sido creado: llevar calma y controlar la agitación dentro de las salas de los hospitales psiquiátricos; perpetuar el tratamiento para las personas que están fuera del manicomio y son consideradas como crónicamente perturbadas; conseguir que los pacientes se muestren indiferentes a aquello que ocurre con ellos tanto dentro, como fuera del hospital psiquiátrico. Dicho de otro modo, la clorpromazina mostró ser un excelente auxiliar para calmar y disciplinar a los pacientes psicóticos dentro y fuera de los manicomios.

La descripción que Delay y Deniker (1952b) hicieron de los pacientes tratados, destacando la aparente indiferencia o demora en responder a estímulos externos, la neutralidad emocional y afectiva, la disminución de la iniciativa será retomada por Deniker en un texto de 1987. Allí, refiriéndose a las investigaciones realizadas en 1957, compara los datos experimentales obtenidos en laboratorio, realizados con ratas, con los relatos clínicos de los pacientes. Resume esos datos en la tabla presentada en la Figura 2, donde sintetiza las características psicofisiológicas de los neurolépticos. 
TABLEAU 8

Caractéristiques psychophysiologiques des neuroleptiques (1957)

\begin{tabular}{l|l}
\hline DONNÉES EXPÉRIMENTALES & DONNÉES CLINIQUES \\
\hline
\end{tabular}

1. Création d'un état d'indifférence psychomotrice spéciale

Hypersomnie réversible par stimulus banals (E.E.G. de sommeil physiologique avec étalement des stades initiaux, rareté des rythmes rapides de veille et abondance d'ondes lentes. Absence des fuseaux).

Diminution de l'actıvité locomotrice spontanée et provoquée.

Inhibition des, eflexes conditionnes et de l'apprentussage.

Tendance à la catalepsie.
Rareté et lenteur des mouvements, hypo- ou amimie.

Indifférence psychique, dimunution de l'inituatave. Neutralite Emotionnelle et affective. Pas d'altérations grossières de la conscience ni des facultés intellectuelles.

\section{Efficacité vis-d̀-vis des états d'excitation et d'agitation}

Tranquillisation des animaux naturellement agressifs.

Action sur la sham-rage des anımaux décortıqués.

Suppression de l'hyperkintsie des "souris tournantes IDPN \# sans narcose ni parésies.

\section{Efficacité vis-à-vas du syndrome maniaque.}

Effet sur l'excitation et l'agitation psychotıques en genéral.

Action sur l'agressivité et l'impulsivité.

Figura 2: Características psicofisiológicas de los neurolépticos (Deniker, 1987, p.48)

La primera característica psicofisiológica identificada en pacientes que usan neurolépticos es la "indiferencia psíquica, la disminución de iniciativas. Sin gran alteración de conciencia", datos que se corresponden en los animales de laboratorio, a la "disminución de la actividad y tendencia a la catalepsia" (Deniker, 1987, p.48).

Si recordamos que esa tendencia a la indiferencia ya había sido identificada por Laborit, cuando se refiere a la indiferencia al dolor, y por Lehmann, cuando se refiere a la indiferencia frente a los delirios, todo parece indicar que el sentimiento de "indiferencia", 
tanto al medio como al tratamiento, es un efecto terapéutico deseado. La indiferencia se asocia a la disminución de agitación, de agresividad, de impulsividad, así como también a la disminución de tentativas de fuga y a la aceptación del tratamiento. Los descubridores de la clorpromazina parecen aproximarse, así, al modo como Foucault (2003, p.268) definía la acción de las drogas psiquiátricas: "Las drogas - esencialmente el opio, el cloroformo, el éter -, eran, por supuesto, como aún lo son las drogas actuales, un instrumento disciplinario evidente, reino el orden, de la calma, de la imposición de silencio".

Así, la pretendida "revolución psicofarmacológica", lejos de representar una ruptura con los procedimientos de choque que precedieron a los neurolépticos, parece establecer una continuidad en lo que se refiere a aquello que, en ambos casos, se consideró como eficacia terapéutica. Ya sea que hablemos de choque por insulina, lobotomía, electrochoque, opio, o éter, ya sea que hagamos referencia al descubrimiento de la clorpromazina, los efectos deseados permanecen idénticos: conquistar la indiferencia, la calma y el silencio necesarios para permitir la gestión de la locura.

\section{AGRADECIMIENTOS}

El presente trabajo recibió apoyo de la Coordenação de Aperfeiçoamento de Pessoal de Nível Superior Brasil (Capes). Código de aporte 001. Proceso n.88881.121008/2016-01 (Capacitación sénior en el exterior).

\section{NOTA}

${ }^{1}$ En esta y en las demas citas literales de textos publicados en otros idiomas la traducción es libre.

\section{REFERENCIAS}

AMARANTE, Paulo; PITTA, Ana; OLIVEIRA, Walter. Direitos humanos e saúde mental. São Paulo: Hucitec, 2017.

APA, American Psychiatric Association. DSM-1. Arlington: APA, 1952.

BAN, Thomas. Fifty years chlorpromazine: a historical perspective. Neuropsychiatric disease and treatment, v.3, n.4, p.495-500, 2007.

BARUK, Henri. Les thérapeutiques psichiatriques. Paris: Presses Universitaires de France, 1972.

BARUK, Henri. La psychopathologie expérimentale. Paris: Presses Universitaires de France, 1967.

BAUMEISTER, Alan. The chlorpromazine enigma. Journal of the History of the Neurosciences, v.22, n.1, p.14-29, 2013.

BREGGIN, Peter. Rational principles of psychopharmacology for therapists, healthcare providers and clients. Journal of Contemporary Psychotherapy, v.46, n.1, p.1-13, 2016.

CALDWELL, Ann. Origins of psychopharmacology from CPZ to LSD. Springfield: Charles C.

Thomas, 1970.
CAPONI, Sandra. Uma sala tranquila: neurolépticos para uma biopolitica da indiferença. São Paulo: Liber Ars, 2019.

CYNOWIEC, Esteban. Análisis epistemológico de la construcción y utilización de modelos animales para el estudio de alteraciones psiquiatricas humanas. Tesis (Doctorado en Ciencias Biológicas) - Universidad de Buenos Aires, Buenos Aires, 2017.

DELAY, Jean; DENIKER, Pierre. Efficacy of tofranil in the treatment of various types of depression: a comparison with other antidepressant drugs. Canadian Psychiatry Association Journal, v.4, p.100-112, 1959.

DELAY, Jean; DENIKER, Pierre. 38 cas de psychoses traitées par la cure prolongée et continue de 4560RP. In: Congrès des Médecins Aliénistes et Neurologistes de France et des Pays de Langue Française, 50., 1952, Luxembourg. Comptes rendus. Paris: Masson, 1952a. p.503-511.

DELAY, Jean; DENIKER, Pierre. Utilisation en therapeutique psychiatrique d'une phenithiazine d'action centrale élective (4560 RP). Annales Médico-Psychologiques, v.110, n.2, p.112-131, $1952 \mathrm{~b}$. 
DELAY, Jean; DENIKER, Pierre;

BOURGUIGNON, André. Extrapyramidal movement disorders during chlorpromazine and reserpine therapy. L'Encéphale, v.45, n.4, p.10931098, 1956.

DENIKER, Pierre. The neuroleptics: a historical survey. Acta Psychiatrica Scandinavica, v.82, p.8387, 1990.

DENIKER, Pierre. From chlorpromazine to tardive dyskinesia: a brief history of the neuroleptics. Psychiatric Journal of the University of Ottawa, v.14, n.1, p.253-259, 1989.

DENIKER, Pierre. Psychopharmacologie: les médicaments et drogues psychotropes. Paris: Ellipses, 1987.

DENIKER, Pierre. Qui a inventé les neuroleptiques? Confrontations Psychiatriques, v.13, p.7-11, 1975.

DENIKER, Pierre; DELAY, Jean. Étude de 300 dossiers de malades psychotiques traités par la chlorpromazine en service fermé depuis 1952. L'Encéphale, v.39, p.528-535, 1956.

FOND, Guillaume. L'éloge de la fuite: hommage à Henri Laborit (1914-1995). Tunisie Medicale, v.91, n.8, p.487-489, 2013.

FOUCAULT, Michel. Le pouvoir psiquiatrique. Paris: Gallimard, 2003.

FREITAS, Fernando; AMARANTE, Paulo. Mad in Brasil: um novo espaço para reflexões e debates sobre medicalização e desmedicalização. In: Amarante, Paulo; Pitta, Ana; Oliveira, Walter (org.). Patologização e medicalização da vida: epistemologia e política. São Paulo: Zagodoni, 2016. p.163-170.

GAUDILLIÈRE, Jean-Paul; THOMS, Ulrike. The development of scientific marketing in the twentieth century research for sales. London: Pickering and Chatto, 2015.

GOTSZCHE, Peter. Psicofármacos que matam y denegación organizada. Barcelona: Los Libros del Lince, 2016.

HEALY, David. The creation of psychopharmacology. Cambridge: Harvard Universty Press, 2002.

HEALY, David. Pioneers in psychopharmacology. International Journal of Neuropsychopharmacology, v.3, n.4, p.351-354, 2000.

HENCKES, Nicolas. Reshaping chronicity: neuroleptics and changing meanings of therapy in French psychiatry, 1950-1975. Studies in
History and Philosophy of Science Part C: Studies in History and Philosophy of Biological and Biomedical Sciences, v.42, n.4, p.434-442, 2011.

KUNZ, Eduard. Henri Laborit and the inhibition of action. Dialogues in Clinical Neuroscience, v.16, n.1, p.113-117, 2014.

LABORIT, Henri. L'hibernation artificielle par moyens pharmacodynamiques et physiques. Presse Medicale, v.59, p.1329, 1951.

LABORIT, Henri; HUGUENARD, Pierre. Un nouveau stabilisateur végétatif (le 4560RP). Presse Medicale, v.60, p.206-208, 1952.

LASKER FOUNDATION. Chlorpromazine for treating schizophrenia, 1957. Disponible en: http://www.laskerfoundation.org/awards/show/ chlorpromazine-for-treating-schizophrenia/. Acceso en: 17 mayo 2021.

LEHMANN, Heinz. Chlopromazine in psychiatric conditions. Canadian Medical Association Journal, v.72, p.91-99, 1955.

LEHMANN, Heinz; HANRAHAN, M. Chlorpromazine: new inhibiting agent for psycomotor excitement and manic states. Archives of Neurology and Psychatry, p.227-237, 1954.

LEHMANN, Heinz; SWAZEY, Judith. Lehmann, Swazey interview. In: Massachusetts Institute of Technology (org.). Chlorpromazine in psychiatry: a study of therapeutic innovation. Cambridge, MA: The Massachusetts Institute of Technology, 1974.

LIEBERMAN, Jeffrey; GOLDEN, Robert; STROUP, Scott. Drugs of the psychopharmacological revolution in clinical psychiatry. Psychiatric Services, v.51, n.10, p.1254-1257, 2000.

LÓPEZ-MUÑOZ, Francisco; ALAMO, Cecilio; CUENCA, Eduardo. Aspectos históricos del descubrimiento y de la introducción clínica de la clorpromazina: medio siglo de psicofarmacología. Frenia, v.2, n.1, p.77-107, 2002.

MAZANA, Javier Sebastián; PEREIRA, J.;

CABRERA, R. Cincuenta años de clorpromazina. Revista Española de Sanidad Penitenciaria, v.4, n.3, p.101-113, 2012.

MISSA, Jean-Noël. La psychopharmacologie et la naissance de la psychiatrie biologique. In: Missa, Jean-Noël; Doron, Claude-Olivier. Les maladies mentales. Paris: PUF, 2008. p.131-147.

MISSA, Jean-Noël. Naissance de la psychiatrie biologique: histoire des traitements des maladies mentales au XXe siècle. Paris: PUF, 2006. 
MONCRIEFF, Joanna. Models of drug action, 2013. Disponible en: https://joannamoncrieff. com/2013/11/21/models-of-drug-action. Acceso en: 17 mayo 2021.

MONCRIEFF, Joanna. The myth of the chemical cure. London: Palgrave MacMillan, 2008.

NOBEL PRIZE. The Nobel Price in Physiology or Medicine 1949: Egas Moniz (Biographical), 1949. Disponible en: https://www.nobelprize.org/ prizes/medicine/1949/moniz/biographical/. Acceso en: 17 mayo 2021.

OVERHOLSER, Winfred. Has chlorpromazine inaugurated a new era in mental hospitals? Journal of Clinical and Experimental Psychopatholy, v.17, n.2, p.197-201, 1956.

PARADA, Carlos. Toucher le cerveau, changer l'esprit. Paris: PUF, 2016.

PIGNARRE, Philippe. Les malheurs des psys: psychotropes et médicalisation du social. Paris: La Découverte, 2006.
ROSE, Nikolas. Our psychiatric future. Cambridge: Polity Press, 2019.

SHORTER, Eduard. A history of psychiatry: from the era of the asylum to the age of Prozac. New York: John Wiley and Sons, 1997.

SWAIN, Glasys. Dialogue avec l'insensé. Paris: Gallimard, 1994.

SWAZEY, Judith. Chlorpromazine in psychiatry: a study of therapeutic innovation. Cambridge, MA: The Massachusetts Institute of Technology, 1974.

WHITAKER, Robert. La psiquiatría defiende sus antipsicóticos: un caso práctico de corrupción institucional. Informe MIA, 2017. Disponible en: https://madinamerica-hispanohablante.org/lapsiquiatria-defiende-sus-antipsicoticos-un-casopractico-de-corrupcion-institucional-robertwhitaker/. Acceso en: 4 sep. 2019.

WHITAKER, Robert. Mad in America: bad science, bad medicine and the enduring mistreatment of the mentally ill. New York: Basic Books, 2010. 